\title{
Frequency of Bacteremia due to Carbapenemase-producing Enterobacteriaceae in Pediatric Hematology-Oncology Can Be Reduced by Measures of Hospital Infection Control
}

\author{
Désirée Caselli ${ }^{1}$, Antonella Colombini ${ }^{2}$, Daniele Zama $^{3}$, Elio Castagnola ${ }^{4}$, Paola Muggeo ${ }^{5}$, Milena La \\ Spina $^{6}$, Katia Perruccio ${ }^{7}$, Raffaella De Santis ${ }^{8}$, Francesca Carraro ${ }^{9}$, Federico Mercolini ${ }^{10}$, Maria Grazia \\ Petris $^{11}$, Daniela Onofrillo ${ }^{12}$, Rossella Mura ${ }^{13}$, Valentina Barretta ${ }^{14}$, Simone Cesaro ${ }^{14}$ \\ ${ }^{1}$ Infectious Diseases, Giovanni XXIII Children Hospital, Azienda Ospedaliero Universitaria Consorziale Policlinico, \\ Bari, Italy \\ ${ }^{2}$ Pediatric Hemato-Oncology, Fondazione MBBM, Milano Bicocca University, San Gerardo Hospital Monza, Italy \\ ${ }^{3}$ Department of Pediatrics, Pediatric Oncology and Haematology Unit "Lalla Seràgnoli", Sant'Orsola Malpighi \\ Hospital, University of Bologna, Bologna, Italy \\ ${ }^{4}$ Infectious Diseases Unit, IRCCS Istituto Giannina Gaslini, Genova, Italy \\ ${ }^{5}$ Pediatric Oncology / Hematology, Azienda Ospedaliero-Universitaria consorziale Policlinico di Bari, Italy \\ ${ }^{6}$ Pediatric Hemato-Oncology Unit, Azienda Policlinico Vittorio Emanuele, Catania, Italy \\ ${ }^{7}$ Pediatric Oncology Hematology, Santa Maria della Misericordia Hospital, Perugia, Italy. \\ ${ }^{8}$ Pediatric Hematology Oncology, Casa Sollievo della Sofferenza Hospital, San Giovanni Rotondo, Italy. \\ ${ }^{9}$ Pediatric Onco-Hematology, Stem Cell Transplantation and Cellular Therapy Division, Regina Margherita Children's \\ Hospital, Torino, Italy \\ ${ }^{10}$ Pediatric Hemato-Oncology Unit, Department of Pediatrics, Regional Hospital of Bolzano, Bolzano, Italy \\ ${ }^{11}$ Dept. Women's and Children's Health, Clinic of Pediatric Hemato-Oncology, University of Padua, Italy \\ ${ }^{12}$ Hematology-Oncology Department, Pediatric Hematology and Oncology Unit, Santo Spirito Hospital, Pescara, Italy \\ ${ }^{13}$ Pediatric Hematology Oncology, Azienda Ospedaliera Brotzu, Cagliari, Italy \\ ${ }^{14}$ Pediatric Hematology-Oncology, Department of Mother and Child, Azienda Ospedaliera Universitaria Integrata, \\ Verona, Italy
}

\begin{abstract}
Objectives: The spread of carbapenemase-producing Enterobacteriaceae (CPE) is an emerging problem in children undergoing cancer-directed chemotherapy. A previous Italian survey reported a threefold increase in CPE colonization rate and a fourfold increase of CPE bloodstream infections in a 2 year-observation time interval.

Methods: To assess the efficacy of the measures put in place to control this emergency, a second survey was performed in the years 2016-2017 and the results were compared.

Results: The overall rate of colonization was comparable between the two study periods: $0.50 \%(2016-2017)$ vs. $0.48 \%$ (2012-2013). MDR/CPE bacteremia was now reported in 13 children from six centers. The rate of MDR/CPE bacteremia was 0.12 for 1,000 days of hospitalization $(95 \% \mathrm{Cl}, 0.07-0.21)$ and compared favorably with that observed in the previous survey $(0.42$ for 1,000 days of hospitalization; $95 \% \mathrm{Cl}, 0.31-0.57 ; p<0.0001)$. The strains responsible for bacteremia were Klebsiella pneumonia in 9/13 children, Escherichia coli in 2/13, Citrobacter spp. and Stenotrophomonas maltophilia in one case each. The antibiotic susceptibility was available for 10 MDR/CPE strains.

Conclusion: The number of patients colonized by CPE remained stable over the years, while the number of bloodstream infections decreased, as well as the resulting mortality. We conclude that children undergoing chemotherapy are at risk for CPE colonization/infection but the awareness of their colonization status may allow reducing CPE morbidity and mortality. J Microbiol Infect Dis 2021; 11(1):27-31.
\end{abstract}

Keywords: Carbapenemase-producing Enterobacteriaceae, children, oncology, hematology, infection control

Correspondence: Dr. Désirée Caselli, Director Infectious Diseases Unit, Pediatric Hospital Giovanni XXIII Azienda OspedalieroUniversitaria consorziale Policlinico di Bari, Bari, Italy

Email: desiree.caselli@policlinico.ba.it

Received: 20 July 2020 Accepted: 08 March 2021

Copyright (C JMID / Journal of Microbiology and Infectious Diseases 2021, All rights reserved 


\section{INTRODUCTION}

Bacteria resistant to most antibiotic classes, including carbapenemase-producing Enterobacteriaceae (CPE), are emerging throughout the world [1]. Since CPE identification in 1993, several carbapenemases have been identified in Enterobacteriaceae [2]. Klebsiella pneumonia carbapenemaseproducing strain $(\mathrm{KPC}+)$ is the single most frequent CPE, first described in 1996, but unfortunately thereafter spreading rapidly worldwide [3]. The rapid spread of CPE, multidrug-resistant (MDR), became a serious threat for patients receiving antineoplastic chemotherapy. In 2015, we reported the results of a nationwide survey among centers participating in the Italian network of pediatric hematology-oncology (Associazione Italiana Ematologia Oncologia Pediatrica; AIEOP). During a 2-year observation period (2012-2013), a threefold increase in the colonization rate, and a fourfold increase of bloodstream infectious episodes caused by CPE was observed, with a 90 -day mortality of $14 \%$. As a result, all centers implemented measures of hospital infection control, including the screening for intestinal MDR strains by rectal swab in every inpatient, on admission [4]. In 2013 the Istituto Superiore di Sanità (ISS) start an Italian surveillance of antibiotic resistance based on a sample of hospital laboratories reporting to the European Antimicrobial Resistance network (EARS-Net), which showed that $K$. pneumoniae is the principal antibiotic-resistance threat in Italy. To monitor and improve the prevention and control of these infections, in February 2013 the Italian Ministry of Health (MoH) instituted national surveillance for CPE through a circular letter (MoH DGPRE $n^{\circ} 4968$ 26/02/2013), asking the 19 regional and the two provincial health authorities to report all cases of BSI due to carbapenem-resistant/-intermediate and/or carbapenemase-producing $K$. pneumoniae or $E$. coli. To control the spread of CPE infections, the circular letter that instituted the CPE surveillance also contained specific recommendations such as the application of infection control protocols including screening of patients and isolation of cases, especially in intensive care and other high-risk wards [5]. Contact precautions include patient placement, gowns/aprons, gloves, patient transport, disposable noncritical patientcare equipment /patient-dedicated use of such equipment, and environmental measures. Active screening of "at-risk" patients on admission to a healthcare setting, encompasses rectal screening, as well as screening from any other site which is either actively infected, e.g. draining wounds, or considered to be colonized [6].

In 2013 AIEOP Centers have started to comply with the AR-ISS directives. In order to monitor the epidemiological trend of MDR/CPE infectious episodes, we performed a second survey and the results are here reported.

\section{METHODS}

The study was a retrospective data collection on a 24-month period (from January 2016 to December 2017); 13 of the 40 Italian pediatric cancer centers of the Associazione Italiana Ematologia Oncologia Pediatrica (AIEOP) network participated in the study. Of them, eight were also included in the previous study.

Multiple drug resistance (MDR), multidrug resistance, or multi-resistance is antimicrobial resistance shown by a species of a microorganism to at least one antimicrobial drug in three or more antimicrobial categories. Carbapenemase-producing Enterobacteriaceae (CPE) are Gram-negative bacteria that are resistant to the carbapenem class of antibiotics, considered the drugs of last resort for such infections.

The following data were collected: a total number of children cared per year, either newly diagnosed or on treatment; type of tumor (leukemia/lymphoma or solid tumor); the total number of hospital days; routine monitoring of MDR/CPE strains on rectal swab; the number of patients colonized by MDR/CPE; the number of patients with bacteremia; cause of bacteremia, and a number of patients who died following bacteremia. Strains were considered as "nonsusceptible" to carbapenems when they tested resistant or non-susceptible according to the interpretative criteria recommended by European Committee on Antimicrobial Susceptibility Testing (EUCAST) [7], or by the Clinical and Laboratory Standards Institute (CLSI) [8]. Incidence of colonization and bacteremia were calculated as rates (episodes/1,000 days of hospital admission) during each year of observation. The mortality of bacteremic patients was evaluated on day 90 
after the first positive blood culture. All the analyses were performed with SAS version 9.4 (SAS Institute Inc., Cary, NC, USA).

\section{RESULTS}

During the study period, the total number of episodes of admission was 8,257: 3,361 (41\%) for patients with solid tumors, and 4,896 (59\%) for patients with leukemia or lymphoma. The median number of patients cared for by each center was 396 (range, 109 to 1,824). A screening program for detection of MDR/CPE carrier was in place in 12 of the 13 (92\%) participating centers during the study period. Table 1 reports data on colonization and bacteremia. Overall, in 53 of the 8,257 episodes $(0.64 \%)$, the patient was found to be carriers of MDR/CPE. This finding accounted for an overall rate of colonization of $0.5 / 1,000$ days of hospitalization $(95 \% \mathrm{Cl}, 0.37-0.65)$. The comparison of the results of the current survey with those of the previous study (table 1) shows that the rate of colonization was comparable between the two study periods: $0.50 \%$ (20162017) vs. $0.48 \% \quad(2012-2013)$. MDR/CPE bacteremia was reported in 13 children from six centers. The rate of MDR/CPE bacteremia was 0.12 for 1,000 days of hospitalization $(95 \% \mathrm{Cl}$, $0.07-0.21$ ) and compared favorably with that observed in the previous survey $(0.42$ for 1,000 days of hospitalization; $95 \% \mathrm{Cl}, 0.31-0.57$; $\mathrm{p}<0.0001$ ).

The number of centers participating in the study was similar: 15 in 2012-2013 vs. 13 in 20162017. When the comparison between the two eras (2012-2013 vs. 2016-2017) was restricted to the eight centers participating in both surveys, no difference was observed.

The strains responsible for bacteremia were Klebsiella pneumoniae in 9/13 children, Escherichia coli in 2/13, Citrobacter spp. and Stenotrophomonas maltophilia in one case each. The antibiotic susceptibility was available for $10 \mathrm{MDR} / \mathrm{CPE}$ strains. All strains were resistant to third-fourth generation cephalosporin, piperacillin/tazobactam, and carbapenems; they were susceptible to amikacin in seven of nine tested, colistin in five of five, fosfomycin in six of seven, and tigecycline in five of eight. Death from any cause occurred in two of the 13 patients with bacteremia (15\%). Both patients, a 6-year old female with severe aplastic anemia, undergoing allogeneic hemopoietic stem cell transplant from a matched unrelated donor, and a 10-year old female with acute myeloid leukemia, had a septic shock by MDR Klebsiella pneumoniae and died. The death occurred after 11 and 15 days from the first positive blood culture, respectively.

Table 1. Epidemiology of CPE colonization and bacteremia in 13 Italian pediatric hematology-oncology centers.

\begin{tabular}{|c|c|c|c|c|c|c|}
\hline \multirow{2}{*}{$\begin{array}{l}\text { Variables } \\
\text { Time interval }\end{array}$} & \multicolumn{3}{|c|}{ Current Study } & \multicolumn{3}{|c|}{ Previous study } \\
\hline & 2016 & 2017 & Total & 2012 & 2013 & Total \\
\hline Colonized patients & 26 & 27 & 53 & 15 & 35 & 50 \\
\hline $\begin{array}{l}\text { Total number of days of } \\
\text { hospitalization }\end{array}$ & 52,420 & 53,709 & 106,129 & 50,513 & 53,431 & 103,944 \\
\hline $\begin{array}{l}\text { Rate of colonization } / 1,000 \text { days of } \\
\text { hospitalization }(95 \% \mathrm{Cl})\end{array}$ & $\begin{array}{l}0.5(0.32- \\
0.73)\end{array}$ & $\begin{array}{l}0.5(0.33- \\
0.73)\end{array}$ & $\begin{array}{l}0.5(0.37- \\
0.65)\end{array}$ & $\begin{array}{l}0.30(0.17- \\
0.49)\end{array}$ & $\begin{array}{l}0.65(0.49- \\
0.61)\end{array}$ & $\begin{array}{l}0.48(0.36- \\
0.63)\end{array}$ \\
\hline Bacteremia (No of episodes) & 6 & 7 & $13^{*}$ & 8 & 36 & 44 \\
\hline $\begin{array}{l}\text { Rate of bacteremia/ } 1,000 \text { days of } \\
\text { hospitalization }(95 \% \mathrm{Cl})\end{array}$ & $\begin{array}{l}0.11(0.04- \\
0.25)\end{array}$ & $\begin{array}{l}0.13(0.05- \\
0.27)\end{array}$ & $\begin{array}{l}0.12(0.07- \\
0.21)\end{array}$ & $\begin{array}{l}0.16(0.07- \\
0.31)\end{array}$ & $\begin{array}{l}0.67(0.47- \\
0.93)\end{array}$ & $\begin{array}{l}0.42(0.31- \\
0.57)\end{array}$ \\
\hline
\end{tabular}

${ }^{*}$ Of the 13 patients with CPE bacteremia, 2 died, one in 2016 and 1 in 2017, due to MDR Klebsiella pneumoniae

\section{DISCUSSION}

The fast and wide spreading of CPE/MDR infections among immune-compromised patients, observed in the last years, represented an excess of "preventable deaths" on which much attention was paid. The results of a survey 
performed in the period 2012-2013 had shown a threatening number of MDR/CPE episodes of bacteremia in children treated for cancer $[9,10]$. This prompted the centers to enforce measures of hospital infection control. They included early identification of MDR/CPE colonized patients by rectal swab screening; adoption of hospital contact precautions by caregivers of colonized patients; single room or cohort isolation for colonized patients; empirical antibiotic therapy guided by the antibiotic susceptibility of colonizing germ; rapid identification of the causative germ of febrile episodes during neutropenia.

To assess the efficacy of such measures, we performed a second, follow-up survey on the years 2016-2017. The differences between the study populations in the two surveys were limited. About one-half of the participating centers were the same in the two studies. All Italian geographical macro-areas were represented in the 2016-2017 survey, with six centers from Northern Italy, three from the center of Italy, and three centers from Southern Italy and islands.

By the time of the follow-up survey, routine screening for colonization of patients was in place in all but one participating center (92\%), while it had been progressively introduced during the years of the initial survey (one-quarter of the centers in 2012, 60\% in 2013) [1]. Overall, the colonization rate observed by routine screening was $0.5 / 1,000$ days of hospitalization, which was not different from what observed in the 2012-2013 survey, although on lower numbers of patients. Interestingly, the MDR/CPE bacteremia rate was significantly lower in 20162017 than in 2012-2013, with 0.12 vs. $0.48 / 1,000$ days of hospitalization $(p<0.0001)$. This difference was confirmed when the analysis was focused on the eight centers which participated in both surveys. Mortality by MDR/CPE remained in the range of $15 \%$ in both studies.

In conclusion, circulation of MDR/CPE strains remained stable in children admitted for cancer chemotherapy in the Italian centers between 2016-2017 compared to 2012-2013, suggesting that the "epidemic" of MDR/CPE, observed at the beginning of the decade, remained then under control. Furthermore, this was associated with a lower number of bacteremia episodes, thus suggesting that higher awareness of this problem may have resulted in a closer application of preventive measures. The analysis of the spectrum of antibiotic susceptibility of isolates from blood cultures showed that amikacin maintains a good efficacy as well as colistin, fosfomycin, and tigecycline. Although some data are available in adult patients with cancer [11-13], our study provides evidence of this problem in children.

\section{ACKNOWLEDGMENTS}

Financial Support: This study was performed without any financial support.

Conflict of interest: The authors have declared no conflicts of interest.

\section{REFERENCES}

1. Tassios GL, Daikos LS, Tzouvelekis A Markogiannakis $M$, Psichogiou PT. Carbapenemases in Klebsiella pneumoniae and other Enterobacteriaceae: an evolving crisis of global dimensions. Clin Microbiol Rev 2012; 25:682-707.

2. Queenan AM, Bush K. Carbapenemases: the versatile b-lactamases. Clin Microbiol Rev 2007; 20:440-58.

3. Tsioutis C, Eichel VM, Mutters NT. Transmission of Klebsiella pneumoniae carbapenemase (KPC)producing Klebsiella pneumoniae: the role of infection control. J Antimicrob Chemother. 2021; 76 (Supplement 1):i4-i11.

4. Caselli D, Cesaro S, Fagioli F, et al. Incidence of colonization and bloodstream infection with carbapenem-resistant Enterobacteriaceae in children receiving antineoplastic chemotherapy in Italy. Infect Dis 2016; 48:152-5.

5. lacchini S, Sabbatucci M, Gagliotti $C$, et al. Bloodstream infections due to carbapenemaseproducing Enterobacteriaceae in Italy: results from nationwide surveillance, 2014 to 2017. Euro Surveill. 2019;24(5):1800159.

6. Magiorakos AP, Burns K, Rodríguez Baño J. et al. Infection prevention and control measures and tools for the prevention of entry of carbapenemresistant Enterobacteriaceae into healthcare settings: guidance from the European Centre for Disease Prevention and Control. Antimicrob Resist Infect Control 6, 113 (2017).

7. Arendrup MC, Hope W, Howard SJ. EUCAST Definitive Document E.Def 9.2 Method for the Determination of Broth Dilution Minimum Inhibitory Concentrations of Antifungal Agents for Conidia Forming Moulds; EUCAST: Basel, Switzerland, 2014. 
8. Clinical Laboratory Standards Institute. Reference Method for Broth Dilution Antifungal Susceptibility Testing of Filamentous Fungi; Approved Standard, 2nd ed.; CLSI document M38-A2; Clinical and Laboratory Standards Institute: Wayne, PA, USA, 2008.

9. Martelius T, Jalava J, Kärki T, et al. Nosocomial bloodstream infections caused by Escherichia coli and Klebsiella pneumoniae resistant to thirdgeneration cephalosporins, Finland, 1999-2013: Trends, patient characteristics and mortality. Infect Dis 2016; 48:229-34.

10. Marín M, Gudiol C, Garcia-Vidal C, Ardanuy C, Carratalà J. Bloodstream infections in patients with solid tumors: epidemiology, antibiotic therapy, and outcomes in 528 episodes in a single cancer center. Medicine (Baltimore). 2014;93(3):143-9.

11. Avendano E, Raman G, Chan J, McCann E. Burden of carbapenem non-susceptible infections in high-risk patients: systematic literature review and meta-analysis. Antimicrob Resist Infect Control 2020; 9: 193.

12. Viale $P$, Tumietto $F$, Giannella M, et al. Impact of a hospital-wide multifaceted programme for reducing carbapenem-resistant Enterobacteriaceae infections in a large teaching hospital in northern Italy. Clin Microbiol Infect 2015; 21(3):242-7.

13. Spyridopoulou K, Psichogiou M, Sypsa V, et al. Containing Carbapenemase-producing Klebsiella pneumoniae in an endemic setting. Antimicrob Resist Infect Control 2020; 9(1):102. 